\title{
IRM avec chélates de gadolinium et fibrose systémique néphrogénique : premier cas clinique décrit avec dosage du gadolinium par ICP-MS
}

\section{Gadolinium MRI and nephrogenic systemic fibrosis : first case report with gadolinium determination by ICP-MS}

\section{Élodie SAUSSEREAU ${ }^{(1) *}$, Christian LACROIX ${ }^{(1)}$, André CATTANEO ${ }^{(2)}$, Loïc MAHIEU ${ }^{(1)}$, Jean-Pierre GOULLÉ(1)}

(1) Laboratoire de Pharmacocinétique et de Toxicologie Cliniques, Groupe Hospitalier du Havre, BP 24 - 76083 Le Havre

(2) Service de Néphrologie-Dialyse, Croix Rouge Française, Hôpital et Institut de Soins Infirmiers 76230 Bois Guillaume

*Auteur à qui adresser la correspondance : Élodie SAUSSEREAU, Laboratoire de Pharmacocinétique et de Toxicologie Cliniques, Groupe Hospitalier du Havre, BP 24 - 76083 Le Havre Tél : 0232733218 - Fax : 0232733238 - E-mail : esaussereau@ch-havre.fr

Ce travail a été présenté partiellement au XV ime Congrès Annuel de la Société Française de Toxicologie Analytique

(Reçu le 14 août 2007 ; accepté après modification le 10 novembre 2007)

\section{$R E ́ S U M E ́$}

La fibrose systémique néphrogénique (FSN) est une pathologie rare, d'étiologie inconnue, observée chez des patients souffrant d'insuffisance rénale sévère. Cette pathologie systémique, dont la morbidité est élevée, affecte principalement la peau. Plusieurs publications récentes évoquent un lien de causalité entre l'exposition aux produits de contraste à base de gadolinium utilisés en imagerie médicale et le développement de la FSN. Nous décrivons un cas de FSN, confirmée par une biopsie cutanée, chez une patiente de 62 ans en insuffisance rénale et sous hémodialyse. De nombreuses

\section{SUMMARY}

Nephrogenic systemic fibrosis (NSF) is a rare disease of unknown cause that affects patients with severe renal failure. This multisystemic disorder with significant morbidity, affects principally the skin. Recent publications suggested an association between exposure to gadolinium-containing contrast agents and subsequent development of NSF. We present one case of NSF histologically confirmed in a 62 year. old woman. This renal insufficiency and dialysis patient underwent numerous magnetic resonance imaging examinations using gadolinium-based contrast media. For the first 
injections de produits de contraste contenant des chélates de gadolinium ont été pratiquées chez cette patiente. Ce cas de FSN est le premier associé à des dosages de gadolinium $\left.{ }^{157} \mathrm{Gd}\right)$ dans le plasma et le sang total, mais également dans les cheveux et les ongles. Les concentrations et teneurs en ${ }^{157} \mathrm{Gd}$, mesurées avec une technique utilisant une torche à plasma couplée à un spectromètre de masse (ICP-MS), sont très élevées. Les résultats obtenus illustrent une nouvelle fois l'association probable entre l'exposition au gadolinium et la fibrose systémique néphrogénique chez des sujets en insuffisance rénale terminale. Le dosage du gadolinium dans les milieux biologiques pourrait avoir un intérêt probant chez les patients insuffisants rénaux ayant nécessité un examen d'imagerie médicale avec injection de gadolinium.

\section{MOTS-CLÉS}

Gadolinium, ICP-MS, IRM, fibrose systémique néphrogénique.

\section{Introduction}

La fibrose systémique néphrogénique (FSN), également connue sous le terme de dermopathie systémique néphrogénique, est une maladie rare décrite pour la première fois dans la littérature en 2000 (1). Environ 200 millions de malades ont reçu une injection de gadolinium depuis 1980 ; seulement un peu plus de 200 cas de FSN ont été rapportés dans le monde (2-4). Cette fréquence est probablement sous-estimée, le syndrome étant connu depuis peu. La FSN est une pathologie systémique, affectant principalement la peau, associée à une morbidité importante. L'évolution clinique est variable, mais la FSN peut parfois entraîner le décès du patient. Cette pathologie, apparentée à la sclérodermie, n'a été observée jusqu'à présent que chez des patients en insuffisance rénale sévère avec un débit de filtration glomérulaire inférieur à $30 \mathrm{~mL} / \mathrm{min} / 1,73 \mathrm{~m}^{2}$, ou chez des sujets ayant eu ou en attente d'une transplantation hépatique (5). La FSN se développe sur une période allant de quelques jours à plusieurs semaines, le plus souvent chez des sujets de plus de 50 ans ; la proportion d'hommes et de femmes étant sensiblement équivalente $(2,6-8)$. La FSN apparaît généralement sous la forme d'un œdème initial des mains et des pieds, suivi d'un épaississement et d'une induration progressive de la peau avec une texture de bois et une augmentation des cellules dermiques fibroblastiques associée au remodelage du collagène et aux dépôts de mucine (6). Les lésions, conséquence d'une fibrose dermique excessive, sont douloureuses, prurigineuses et souvent le siège de troubles de la pigmentation.

L'épaississement empêche fréquemment la flexion et l'extension des articulations, résultant en de sévères contractures qui peuvent altérer la mobilité. Ces lésions progressent ensuite vers les racines des membres à un time, gadolinium $\left({ }^{157} \mathrm{Gd}\right)$ determination was performed in plasma and whole blood, as well as in hair and fingernails. Large gadolinium contents were measured in all specimens by inductively coupled plasma mass spectrometry (ICP-MS). The results observed are a new illustration of a causative role of gadolinium in the pathophysiology of NSF in endstage-renal-disease patients. Gadolinium determination in biological fluids could be of major interest for renal insufficiency patients whose diagnostic required gadoliniumenhanced magnetic resonance imaging.

\section{KEY-WORDS}

Gadolinium, ICP-MS, MRI, nephrogenic systemic fibrosis.

rythme variable, et sont généralement distribuées symétriquement sur les membres et le tronc. L'extrémité cervico-céphalique est rarement intéressée (6). La fibrose associée à la FSN peut s'étendre au-delà du derme et atteindre les tissus sous-cutanés, les muscles striés, le diaphragme, la plèvre, le péricarde et le myocarde (6). Cette pathologie, d'étiologie encore inconnue, est possiblement multifactorielle. Depuis 2006, un lien de causalité a été évoqué entre l'injection de produits de contraste contenant du gadolinium (Gd) et la FSN $(2,3,6-10)$. Le nombre de cas décrits de FSN a augmenté parallèlement à l'utilisation de plus en plus fréquente, dans le domaine de l'imagerie médicale, des produits de contraste contenant du gadolinium (2). Le gadolinium $\mathrm{Gd}^{3+}$ est un ion métallique appartenant à la famille des terres rares. Il possède 7 électrons célibataires à l'origine d'un effet paramagnétique élevé utilisé en imagerie par résonance magnétique (IRM). Dans le cadre de l'imagerie médicale, le Gd est utilisé sous forme de chélates, molécules plus stables, hydrophiles, prévenant la précipitation du $\mathrm{Gd}^{3+}$ et masquant ainsi sa toxicité. Ces produits de contraste, utilisés par voie intraveineuse, ont une diffusion extracellulaire rapide et sont éliminés essentiellement par filtration glomérulaire sous forme inchangée. La demi-vie d'élimination de ces chélates augmente par conséquent, proportionnellement à la sévérité de l'insuffisance rénale (11-14). La demi-vie du gadodiamide (Omniscan ${ }^{\circ}$ ) est de 1,3 heures chez le sujet sain, de 34,3 heures chez le sujet en insuffisance rénale terminale, de 2,6 heures chez un patient hémodialysé, et atteint 52,7 heures dans le cas d'une dialyse péritonéale (13). En ce qui concerne le gadopentate de diméglumine (Magnevist" ${ }^{\text {tw }}$ ), la demi-vie d'élimination est de 1,5 à 2 heures chez un sujet dont la clairance de la créatinine $\left(\mathrm{Cl}_{\mathrm{CR}}\right)$ est de 60 à $80 \mathrm{~mL} / \mathrm{min}$; elle est de 4 heures en cas d'insuffisance rénale modé- 
rée $\left(\mathrm{Cl}_{\mathrm{CR}}: 40-60 \mathrm{~mL} / \mathrm{min}\right)$ et atteint 10 heures en cas d'insuffisance rénale sévère $\left(\mathrm{Cl}_{\mathrm{CR}}: 20-40 \mathrm{~mL} / \mathrm{min}\right)$ (14). Chez le sujet hémodialysé, $78 \%$ de la dose de gadopentate de diméglumine administrée par voie intraveineuse, sont éliminés après la première séance d'hémodialyse ; 4 séances sont nécessaires pour une élimination totale du chélate (15). La principale hypothèse expliquant potentiellement le rôle du gadolinium dans la FSN est la transmétallation : dissociation du complexe formé entre le chélate et $\mathrm{Gd}^{3+}$, qui est la forme toxique du gadolinium $(2,4,6-8,16)$. En effet, $\mathrm{Gd}^{3+}$ bloque le système réticulo-endothélial, entre en compétition avec les systèmes calcium-dépendants, ayant pour conséquence des effets neurotoxiques, anticoagulants et sur la contractilité cardiaque. Le gadolinium $\mathrm{Gd}^{3+}$ pourrait agir après captation par les macrophages, par l'intermédiaire de la sécrétion de cytokines préfibrotiques et/ou de facteurs de croissance agissant sur les fibrocytes qui se déposeraient dans les zones de traumatisme, d'inflammation ou de stase (6). Ce mécanisme de transmétallation serait favorisé par des cations endogènes (zinc, fer, cuivre, calcium), ainsi que par l'acidose métabolique fréquente chez les insuffisants rénaux (6). Les lésions vasculaires pourraient également être associées au développement de la FSN (1). Une biopsie cutanée est nécessaire pour l'établissement définitif du diagnostic de FSN.

\section{Observation}

Un cas de fibrose systémique néphrogénique a été observé chez une patiente de 62 ans, hémodialysée depuis 1999 suite à une binéphrectomie en raison d'un adénocarcinone bilatéral rénal. La surveillance oncolo- gique semestrielle de cette patiente a été initialement effectuée avec des scanners thoraco-abdominaux. A partir de 2003, ce suivi oncologique a été poursuivi par IRM en raison d'une allergie sévère avec œdème de Quincke aux produits de contraste iodés. Cette patiente a reçu 12 injections de produits de contraste contenant du gadolinium sur une période d'environ 3 ans (mai 2003-juillet 2006) (Tableau I). Les produits de contraste utilisés chez cette patiente étaient du gadodiamide (Omniscan ${ }^{1}$ ), du gadotérate de méglumine $\left(\right.$ Dotarem $\left.^{\oplus}\right)$ et du gadopentétate de diméglumine (Magnevist ${ }^{*}$ ). En novembre 2004, une dizaine de jours après une injection de gadodiamide, la patiente a présenté, au niveau des membres inférieurs, une perte de sensibilité avec infiltration douloureuse de la peau, une raideur des articulations avec impossibilité de se mettre en génuflexion et une abolition des réflexes ostéo-tendineux. Après une nouvelle injection de gadolinium en décembre 2004 (Magnevist $^{\circledR}$ ), la symptomatologie a évolué avec des difficultés à la marche, les articulations des membres inférieurs devenant de plus en plus raides avec difficultés croissantes pour la flexion des genoux et des pieds. En ce qui concerne les lésions cutanées, l'infiltration de la peau à l'origine d'une texture cartonnée et d'un aspect " peau d'orange » (Figure 1), a progressé vers les membres supérieurs. Le tronc, l'abdomen et le visage n'étaient pas atteints à ce stade de la fibrose. Une coloration brunâtre, violacée de la peau est apparue au niveau des lésions des membres (Figure 2). Après deux injections supplémentaires de gadolinium en février et mai 2005 (Magnevist"), une sclérose très extensive des quatre membres a été observée, prédominant nettement au niveau des membres inférieurs et d'évolution centripète, de la distalité vers l'abdomen. La biopsie cutanée réalisée en décembre 2005, soit 13

Tableau I : Chronologie des IRM pratiquées chez la patiente entre mai 2003 et juillet 2006.

\begin{tabular}{|c|c|c|c|}
\hline Date & Type d'IRM & Produit de Contraste Injecté & Contexte Médical \\
\hline 26 mai 2003 & IRM vertébrale & Omniscan & Douleurs lombaires \\
\hline 5 juin 2003 & Angio-IRM & Dotarem & Angioplastie de l'abord vasculaire (hémodialyse) \\
\hline 23 octobre 2003 & IRM abdomino-pelvienne & Dotarem $^{30}$ & Surveillance oncologique \\
\hline 30 octobre 2003 & Angio-IRM & Dotarem $^{23}$ & Angioplastie de l'abord vasculaire \\
\hline 18 mars 2004 & Angio-IRM & Dotarem & Angioplastie de l'abord vasculaire \\
\hline 10 novembre 2004 & IRM abdomino-pelvienne & Magnevist & Surveillance oncologique \\
\hline 17 novembre 2004 & IRM cérébrale & Omniscan $^{38}$ & Surveillance post-AVC ${ }^{(2)}$ \\
\hline 17 décembre 2004 & IRM cérébrale & Magnevist & Surveillance post-AVC \\
\hline 21 février 2005 & IRM cérébrale & Magnevist & Surveillance post-AVC \\
\hline 30 mai 2005 & IRM cérébrale & Magnevist & Surveillance post-AVC \\
\hline 4 février 2006 & IRM cérébrale & Omniscan & Surveillance post-AVC \\
\hline 20 juillet 2006 & IRM cérébrale & Omniscan $^{2}$ & Surveillance post-AVC \\
\hline
\end{tabular}

(1) Angiographie par résonance magnétique ; ${ }^{(2)}$ Accident vasculaire cérébral 
mois après l'apparition des premiers signes cliniques, a mis en évidence une fibrose compatible avec une sclérose cutanée diffuse spécifique dans le cadre d'une fibrose systémique néphrogénique. Le traitement de 6 mois avec des corticoïdes $(0,5 \mathrm{mg} / \mathrm{kg} / \mathrm{j})$ n'a eu aucune efficacité. La patiente présentait également des troubles auriculo-ventriculaires probablement associés à la FSN $(2,3,6)$. En septembre 2006 , suite à la $12^{\text {ime }}$ IRM (juillet 2006), des dosages de gadolinium ont été réalisés afin d'établir un éventuel lien de causalité entre l'exposition répétée de la patiente au gadolinium et le développement de la FSN. Le rôle du gadolinium dans la physiopathologie de la FSN étant évoqué depuis janvier 2006 (6).

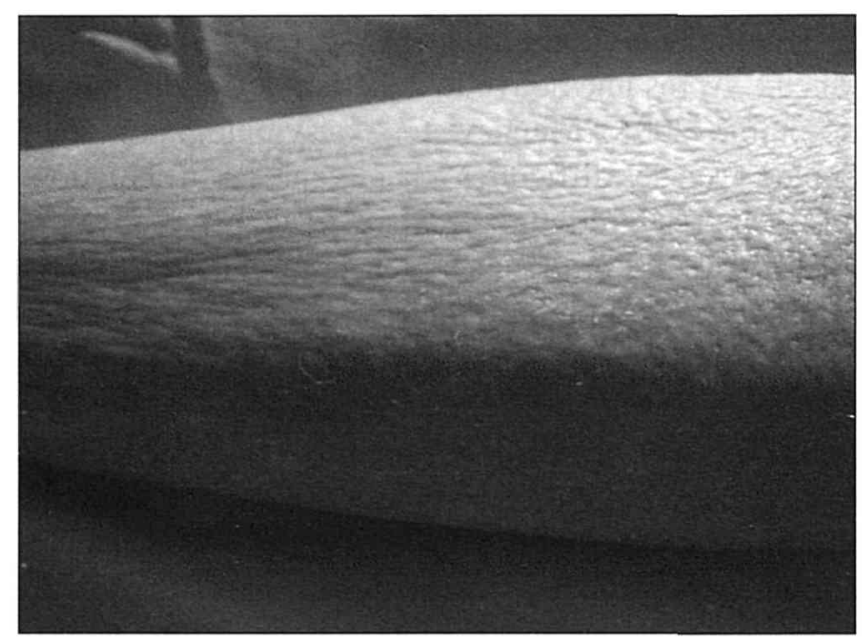

Figure 1 : Aspect "peau d'orange" des lésions cutanées, associé à l'épaississement et à l'induration progressifs de la peau.

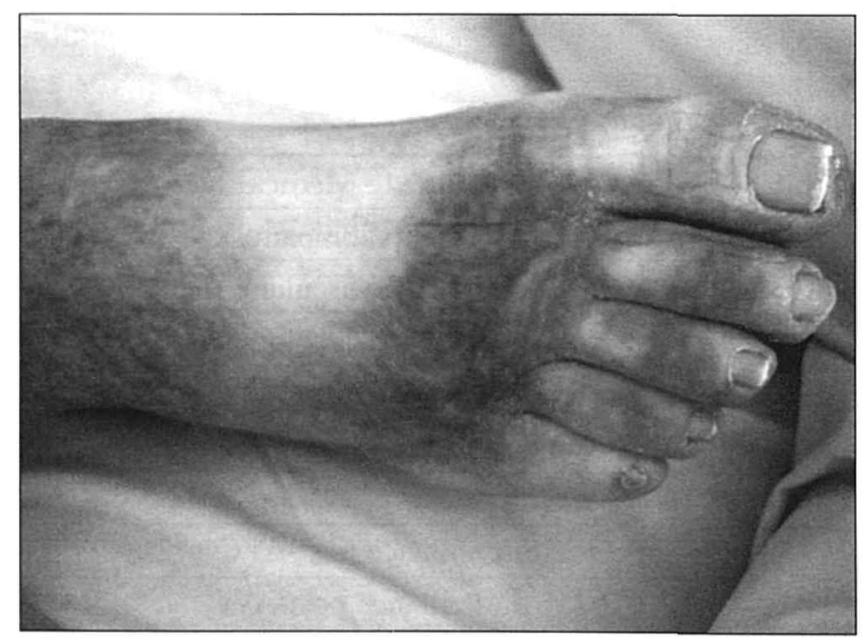

Figure 2 : Coloration brînatre, violacée des lésions cutanées, associée à des troubles de la pigmentation.

\section{Matériel et méthodes}

Le dosage du gadolinium $\left({ }^{157} \mathrm{Gd}\right)$ a été effectué dans deux échantillons sanguins (sang total et plasma) prélevés à 3 mois et demi d'intervalle ; le premier prélèvement ayant été réalisé 3 mois après la dernière IRM. Des dosages ont également été effectués dans une mèche de cheveux de $7 \mathrm{~cm}$ prélevée deux mois après la dernière IRM, ainsi que dans un échantillon unguéal obtenu avec le second échantillon sanguin. Les dosages sont effectués par ICP-MS avec une technique de dosage multiélémentaire des métaux et métalloïdes appliquée au ${ }^{157} \mathrm{Gd}$ (17). Les réactifs, de qualité suprapure pour analyse de traces et la solution étalon proviennent de chez Merck (Darmstadt, Allemagne) et CPI (Amsterdam, Hollande). Cette méthode de dosage multiélémentaire déjà décrite a également été appliquée au dosage $\mathrm{du}{ }^{157} \mathrm{Gd}$ dans les ongles (18). Après décontamination par de l'acétone et de l'eau tiède, $20 \mathrm{mg}$ d'ongles sont minéralisés par $200 \mu \mathrm{L}$ d'acide nitrique suprapur pendant une heure à $70^{\circ} \mathrm{C}$. Après refroidissement, à $100 \mu \mathrm{L}$ de la solution acide obtenue, sont ajoutés $100 \mu \mathrm{L}$ d'acide nitrique à $2 \%$ et $3800 \mu \mathrm{L}$ de diluant (butanol 0,5\%, acide nitrique $1 \%$, triton $0,01 \%$; étalons internes : In et $\mathrm{Rh} 1 \mathrm{ppb}$ ). L'étalonnage est réalisé dans de l'eau surchargée à $0,005-0,01-0,03-0,06$ $0,12-0,24 \mu \mathrm{g}$ de gadolinium par litre. Les mesures ont été réalisées avec une torche à plasma de type ThermoElectron X Series couplée à un spectromètre de masse, modèle X7/CCT (ThermoElectron, Courtaboeuf, France). L'appareil est équipé d'une torche en quartz de 1,5 mm, d'un nébuliseur concentrique en verre borosilicaté de $1 \mathrm{~mL}$ (type concentrique de marque Glass Expansion - Référence ThermoElemental 1201318) avec un débit d'échantillon de $0,85 \mathrm{~mL} / \mathrm{min}$, d'une chambre de nébulisation en quartz munie d'un refroidisseur à effet Peltier régulant sa température à $3^{\circ} \mathrm{C}$, et d'un passeur d'échantillons de type CETAC ASX-510. L'ensemble des données est enregistré sur une station informatique dotée du logiciel d'analyse PlasmaLab version 2.0 sous Windows NT.

\section{Résultats et discussion}

Dans le premier prélèvement sanguin, réalisé 3 mois après la dernière injection de gadolinium, les concentrations plasmatiques et dans le sang total de ${ }^{157} \mathrm{Gd}$ sont respectivement de 11,60 et $36,30 \mu \mathrm{g} / \mathrm{L}$. Ces concentrations sont très élevées, plus de 100 fois la valeur supérieure observée chez 50 sujets non exposés $(0,10 \mu \mathrm{g} / \mathrm{L})$. Trois mois et demi plus tard, les concentrations plasmatiques et dans le sang total qui sont identiques, sont encore importantes, soit $1,80 \mu \mathrm{g} / \mathrm{L}$. La différence 
observée dans le premier prélèvement sanguin, entre la concentration plasmatique et celle obtenue dans le sang total, est difficile à interpréter. En effet, ce cas de FSN est le premier pour lequel des dosages sanguins de gadolinium ont été effectués. D'autre part, la patiente a reçu récemment, en mai 2007, une nouvelle injection de gadolinium; dans le prélèvement sanguin alors effectué, la concentration plasmatique de ${ }^{157} \mathrm{Gd}$ est plus élevée que dans le sang total. Dans la mèche de cheveux, fragmentée en segments de $1 \mathrm{~cm}$, les concentrations de ${ }^{157} \mathrm{Gd}$ varient de 30 à $1.090 \mathrm{pg} / \mathrm{mg}$. L'analyse des cheveux a permis d'observer une teneur en ${ }^{157} \mathrm{Gd}$ plus faible au niveau des pointes, le fragment correspondant probablement à la période de février-mars 2006 (Figure 3). Ce résultat pourrait être expliqué par l'intervalle de 9 mois séparant l'injection de gadolinium de mai 2005 et celle de février 2006. Cette concentration de $30 \mathrm{pg} / \mathrm{mg}$ de ${ }^{157} \mathrm{Gd}$ reste cependant élevée par comparaison à la médiane mesurée chez 50 sujets non exposés $(1 \mathrm{pg} / \mathrm{mg})$. L'augmentation de la teneur en ${ }^{157} \mathrm{Gd}$ dans les deux segments suivants (avrilmai 2006) pourrait être consécutive à l'injection de gadolinium de février 2006. Dans les quatre derniers segments, jusqu'à la racine, la concentration de ${ }^{157} \mathrm{Gd}$ apparaît relativement constante, ce qui pourrait être associé à l'injection supplémentaire de gadolinium pratiquée en juillet 2006. L'interprétation des résultats obtenus à partir de la mèche de cheveux est cependant difficile en l'absence de données dans la littérature scientifique. En ce qui concerne l'échantillon unguéal de $1 \mathrm{~mm}$ prélevé à l'extrémité distale des ongles de la main, la teneur en ${ }^{157} \mathrm{Gd}$ mesurée est très élevée, $1.230 \mathrm{ng} / \mathrm{mg}$. La valeur médiane obtenue chez 130 sujets sains, non exposés au gadolinium, est de $1 \mathrm{pg} / \mathrm{mg}$ (5 $5^{\text {inc }}$ percentile : $0,3 \mathrm{pg} / \mathrm{mg} ; 95^{\text {ime }}$ percentile : 6,6 $\mathrm{pg} / \mathrm{mg}$ ). L'intervalle de 6 mois existant entre la dernière injection de gadolinium et le prélèvement d'ongles, ainsi que la durée de renouvellement des ongles de la main qui est de 6 mois, pourraient expliquer la concen-

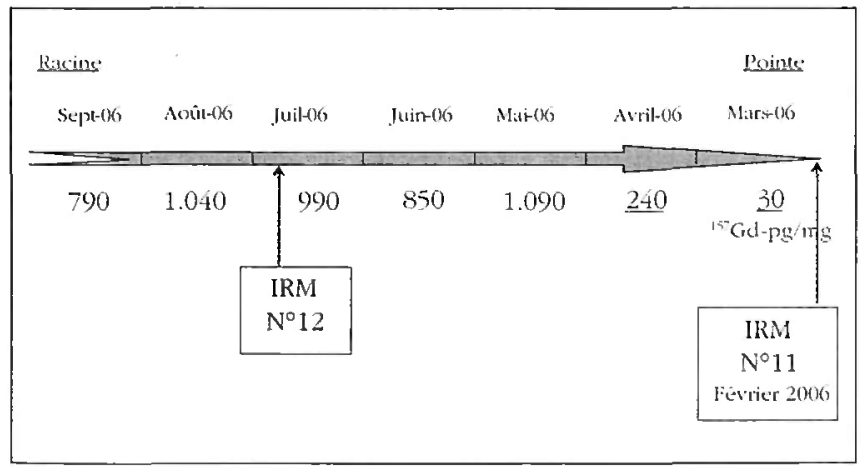

Figure 3 : Teneurs en ${ }^{157} \mathrm{Gd}$ des segments de cheveux prélevés deux mois après la demière injection de gadolinium. tration élevée de ${ }^{157} \mathrm{Gd}$ observée chez la patiente. Ce cas de fibrose systémique néphrogénique est, à notre connaissance, le premier cas pour lequel des dosages de gadolinium ont été effectués, que ce soit dans le plasma, sang total ou autres milieux biologiques (cheveux, ongles). Les résultats précédemment exposés ont été récemment complétés par un dosage de ${ }^{157} \mathrm{Gd}$ dans un fragment de tissu cutané prélevé en juillet 2007, après une nouvelle injection de gadolinium. Une biopsie hépatique réalisée en postmortem (septembre 2007) a permis également la réalisation du dosage de ${ }^{157} \mathrm{Gd}$ dans un fragment de tissu hépatique. Les concentrations observées dans ces deux tissus, cutané et hépatique, sont très élevées, respectivement 20,5 et $119,4 \mathrm{ng} / \mathrm{mg}$ de tissu frais. Ces derniers résultats confirment une importante accumulation de gadolinium chez cette patiente. Cependant, l'absence de référence chez les patients insuffisants rénaux, exposés aux produits de contraste à base de gadolinium et présentant une FSN, est à l'origine d'une interprétation délicate des résultats obtenus. Par conséquent, il serait intéressant de réaliser des dosages de ${ }^{157} \mathrm{Gd}$, dans les différents milieux biologiques (plasma, sang total, urine, phanères), dans une cohorte de patients insuffisants rénaux souffrant de FSN après exposition au gadolinium. Ces études toxicologiques permettraient de disposer de valeurs de référence dans cette population de malades exposés aux différents produits de contraste contenant du gadolinium. Actuellement, les cas de FSN décrits sont associés, en grande majorité, à une exposition au gadodiamide (Omniscan ${ }^{\circledR}$ ). Quelques cas de FSN observée après une injection de gadopentate de diméglumine (Magnevist ${ }^{\text {tix }}$ ) ou de gadoversétamide (Optimark ${ }^{\text {ii }}$ ) ont été rapportés $(5,8,19)$; le rôle des autres produits de contraste à base de gadolinium dans le développement de cette pathologie n'est pas clairement démontré $(3,8)$. Il est intéressant de noter que notre patiente, exposée à trois produits de contraste différents (Omniscan ${ }^{\circledR}$, Dotarem ${ }^{*}$, Magnevist ${ }^{\circledR}$ ), a présenté les premiers signes cliniques associés à la FSN après une injection de gadodiamide. Les manifestations cliniques ultérieures ont été rythmées par trois injections de gadopentate de diméglumine, confirmant l'implication de ce chélate dans la genèse de la FSN. Enfin, il semble important de développer les études pharmacocinétiques, peu nombreuses $(4,20)$, chez des patients en insuffisance rénale exposés au gadolinium, atteints ou non d'une FSN, afin d'évaluer l'efficacité de séances d'hémodialyse consécutives sur le traitement ou la prévention de la fibrose systémique néphrogénique. 


\section{Conclusion}

La fibrose systémique néphrogénique est une pathologie rare, d'évolution rapide, pouvant être légère à invalidante, voire exceptionnellement mortelle. Elle est observée chez des sujets insuffisants rénaux et en acidose, ayant été exposés à des produits de contraste à base de gadolinium. Le nombre de cas de FSN décrits a augmenté parallèlement à l'utilisation de plus en plus fréquente de l'IRM et des produits de contraste contenant du gadolinium; les doses injectées en routine ayant également augmenté. Le cas clinique décrit, qui est le premier cas où des dosages sanguins, capillaires et unguéaux ont été réalisés, semble confirmer ce lien de causalité entre une exposition au gadolinium et la FSN chez les sujets atteints d'insuffisance rénale chronique. De plus, notre patiente a subi de nombreuses IRM et a donc été exposée à de très fortes doses de gadolinium. Ainsi, cette nouvelle observation de FSN pose le problème de la pratique de l'IRM avec ces produits de contraste à base de gadolinium chez les sujets insuffisants rénaux chroniques.

\section{Références}

1. Cowper S. E., Robin H. S., Steinberg S. M., Su L. D., Gupta S., LeBoit P. E. Scleromyxedema-like cutaneous diseases in renal-dialysis patients. Lancet. 2000 ; 356 : 1000-1.

2. Marckmann P., Skov L., Rossen K., Dupont A., Damholt M. B., Heaf J. G., Thomsen H. S. Nephrogenic systemic fibrosis: suspected etiological role of gadodiamide used for contrast-enhanced magnetic resonance imaging. J. Am. Soc. Nephrol. $2006 ; 17: 2359-62$.

3. Sadowski E. A., Benett L. K., Chan M. R., Wentland A. L., Garett A. L., Garett R. W., Djamali A. Nephrogenic systemic fibrosis: risk factors and incidence estimation. Radiology. 2007 ; 243 : 148-57.

4. Broome D. R., Girguis M. S., Baron P. W., Cottrel A. C., Kjellin I., Kirk G. A. Gadodiamide-associated nephrogenic systemic fibrosis: why radiologist should be concerned. Am. J. Roentgenol. 2007 ; 188 : 586-92.

5. Public health advisory: gadolinium-containing contrast agents for magnetic resonance imaging (MRI)_Magnevist, MultiHance, omniscan, OptiMARK, ProHance. U.S Food and Drug Administration.

www.fda.gov/cder/drug/advisory/gadolinium_agents.htm Published June 8, 2006. Updated December, 2006 and May, 2007.

6. Grobner T. Gadolinium-a specific trigger for the development of nephrogenic fibrosing dermopathy and nephrogenic systemic fibrosis? Nephrol. Dial. Transplant. $2006 ; 21$ : 1104-08.
7. Khurana A., Runge V. M., Narayanan M., Greene J. F. Jr., Nickel A. E. Nephrogenic systemic fibrosis. A review of 6 cases temporally related to gadodiamide injection (Omniscan). Invest. Radiol. 2007 ; 42 : 139-45.

8. Thomsen H. S. Nephrogenic systemic fibrosis: a serious late adverse reaction to gadodiamide. Eur. Radiol. 2006 ; $16: 2619-21$.

9. Grobner T., Prischl F.C. Gadolinium and nephrogenic systemic fibrosis. Kidney Inter. $2007 ; 72$ : 260-64.

10. Lim Y.L., Lee H.Y., Low S.C., Chan L.P., Goh N.S., Pang S.M. Possible role of gadolinium in nephrogenic systemic fibrosis: report of two cases and review of the literature. Clin. Exp. Dermatol. 2007 ; 32 : 353-58.

11. Thomsen H.S. Guidelines for contrast media from the European Society of Urogenital Radiology. AJR Am. J. Roentgenol. 2003 ; 181 : 1463-71.

12. Morcos S.K., Thomsen H.S., Webb J.A. Dialysis and contrast media Eur. Radiol. 2002 ; 12 : 3026-30.

13. Joffe P., Thomsen H.S., Meusel M. Pharmacokinetics of gadodiamide injection in patients with severe renal insufficiency and patients undergoing hemodialysis or continuous ambulatory peritoneal dialysis. Acad. Radiol. $1998 ; 5(7): 491-502$.

14. Schuhmann-Giampieri G., Krestin G. Pharmacokinetics of Gd-DTPA in patients with chronic renal failure $1991 ; 26(11): 975-79$.

15. Okada S., Katagiri K., Kumazaki T., Yokoyama H. Safety of gadolinium contrast media agent in hemodialysis patients. Acta. Radiologica. $2001 ; 42$ : 339-41.

16. Boyd A. S., Zic J. A., Abraham J. L. Gadolinium deposition in nephrogenic fibrosing dermopathy. J. Am. Dermatol. $2007 ; 56: 27-30$.

17. Goullé J. P., Mahieu L., Casternant J., Neveu N., Bonneau L., Laine G., Bouige D., Lacroix C. Metal and metalloid multi-elementary ICP-MS validation in whole blood, plasma, urine and hair. Reference values. Forensic Sci. Int. $2005 ; 153: 39-44$

18. Goullé J.P., Mahieu L., Saussereau E., Bouige D., Groenwont S., Lacroix C. Validation d'une technique de dosage multiélémentaire des métaux et métalloïdes dans les ongles par ICP-MS. Valeurs usuelles chez 130 sujets volontaires. Ann. Toxicol. Anal. 2007 ; 19 : 125-34.

19. Thomsen H.S., Morcos S.K., Dawson P. Is there a causal relation between the administration of gadolinium based contrast media and the development of nephrogenic systemic fibrosis (NSF)? Clin. Radiol. 2006 ; 61(11) : 9056.

20. Saitoh T., Hayasaka K., Tanaka Y., Kuno T., Nagura Y. Dialyzability of gadodiamide in hemodialysis patients. Radiat. Med. 2006 ; 24 : 445-51. 